\title{
Understanding narratives with argumentation
}

\author{
Floris BEX ${ }^{\mathrm{a}}$, Trevor BENCH-CAPON ${ }^{\mathrm{b}}$ \\ a Information and Computing Sciences, Utrecht University, The Netherlands \\ ${ }^{\mathrm{b}}$ Computer Science, University of Liverpool, UK
}

\begin{abstract}
In this paper, we show two important connections between computational models of narrative and computational models of argumentation. First, we show how argumentation techniques can be applied to enrich story understanding, especially where an understanding the story requires understanding of the motives of its characters. This also helps to explain how stories can themselves be seen as as arguments for a particular ordering on values within a value based argumentation framework. We illustrate our discussion using biblical parables, taking as our main example the parable of the Good Samaritan.
\end{abstract}

Keywords. narratives, parables, value-based argumentation

\section{Introduction}

We often persuade not by imparting facts and rules, but by providing an interesting narrative, particularly when trying to convince others to adopt particular values and attitudes. In this paper we show how current computational argument techniques can be used to enrich story understanding, and how these techniques show that stories can sometimes function as arguments.

A central concept from research on story understanding is scripts [8], coherent scenarios about common situations such as visiting a restaurant. Scripts still play an important part in computational and cognitive approaches of story understanding (e.g. [7]) and have been applied in, e.g. case-based reasoning, scenario-based evidence analysis and narrative generation.

In our opinion, purely script-based approaches are not, however, best suited to understanding persuasive stories concerning values, such as parables. Scripts represent the way in which we expect typical situations to play out: the more a story adheres to a familiar script, the more plausible a story is considered to be. However, many memorable stories such as parables depend on a twist in the story, something which is out of the ordinary and which challenges conventional attitudes [4]. For example, no-one expects a father to organise a feast for a son who has spent all of his money on wild living (The Prodigal Son (Luke 15:11-32)). Interesting stories often include conflicting attitudes: in the Prodigal Son, the son's older brother wants to turn away his sibling while the father wishes to forgive and welcome his son. In models based on scripts, in which stories are rendered only as causal sequences, these conflicts between characters' values cannot be readily articulated and so remain largely implicit and unexplained. 
Computational argumentation techniques allow us to add a more fine-grained psychological dimension to the causal narrative, in which conflicts between characters' attitudes and challenges to common attitudes can be modelled. This gives us an internal perspective that allows us to represent the deliberations of the characters involved, which in turn allows for a much more subtle analysis of character motive and attitude than we can perform with just the external causal perspective. This analysis may also persuade an audience to adopt a different attitude themselves.

Our model for story understanding, which is explained in section 3 by means of the parable of the The Good Samaritan (Luke 10:25-37), is based on value-based practical reasoning as described in [1]. Stories can be represented as (causal) state transition diagrams, where the transitions represent possible actions by the characters in the story. Character motives are represented by indicating which values are promoted or demoted by the actions in the story. We can then generate practical reasoning arguments of the form I should perform Action because it promotes Value and I should not perform Action because it demotes Value from the diagram. By adding separate arguments denoting the possible attitudes of characters as value orderings, we can construct an Extended Argumentation Framework (EAF) [5] with value preferences attacking attacks, giving a set of (possibly conflicting) arguments representing character choices and attitudes. We can then infer attitudes from the choices made in the story.

This approach to understanding the attitudes represented in a story, allows stories to themselves be used as arguments. Sometimes the conclusion the audience is invited to draw from the story is explicitly mentioned in the text: in fables the moral of the story is usually explicitly included. In other cases the conclusion may depend on the context in which the story is told. The Prodigal Son, for example, is told as a response to the Pharisees remark that he 'welcomes sinners and eats with them' (Luke 15:2); thus the parable can be seen as a justification of fraternising with sinners. Note that the conclusion of the argument is not advice to behave in a particular way, but rather to adopt an outlook, a set of attitudes. In section 3.1 we show how a particular story interpreted by means of an EAF can be used as an argument in a particular dialogical context, using [6]'s extended TPI-protocol for argumentative dialogue to argue for a change in value preferences in a dialogical setting.

\section{Motivating example: The Good Samaritan}

Stories can be a powerful vehicle of persuasion. A story does not persuade by imparting explicit rules, but by exposing a coherent narrative aimed at changing or reinforcing attitudes, so that the stories exemplify various group cultural norms. Many folktales are of this type, as are parables, both secular and biblical. As our example we will use the well-known parable, The Good Samaritan (Luke 10:25-37):

Leading up to the parable, a lawyer asks two questions. The first, "what shall I do to inherit eternal life?", receives an answer justified by scriptural authority, whereas the second, "Who is my neighbour?", is met simply by a story. A traveller is attacked and left wounded by the roadside. Two respectable citizens, a priest and a Levite, pass him by before a Samaritan (a member of a generally despised group) helps him. The lawyer is asked who was neighbour to the wounded man, and answers that it was the Samaritan.

Here the story is used as an argument to justify an answer to a question. It is not meant as a theoretical argument: the aim is not that the lawyer should believe that the 
Samaritan is his neighbour. Nor is the lawyer intended to set out to assist wounded travellers on the road from Jerusalem to Jericho. Unlike practical reasoning proper, there is no specific situation, with a specific choice of actions to resolve. Rather the argument is intended to convince the lawyer (and ultimately of course the audience) to become a different person.

So how exactly does the story convince its audience to change their ways? Govier and Ayers [4] have explored this question in relation to the Good Samaritan, reconstructing it as an argument with five premises (two implicit and added by them) and a normative conclusion. We, however, contend that the claim of the parable should not be a norm such as in certain situations you should do this, but rather an invitation to adopt different attitudes, to be like the Samaritan. To enable a story to have this effect we need a detailed account of the reasoning of the three characters, to articulate the differences in attitude between them, and so identify the attitudes we are being urged to abandon and adopt.

\section{Understanding stories using value-based argumentation}

We apply techniques from computational argumentation to understand the story. We start from [1], which we used previously to capture abductive reasoning in which stories served as explanations of evidence [2]. This model has three main elements: (i) ActionBased Alternating Transition Systems with Values (AATS+V) for encapsulating stories; (ii) arguments based on the Practical Reasoning Argumentation Scheme (PRAS), to generate arguments concerning the individual choices a story character can make; and (iii) Value-based Argumentation Frameworks (VAF), representing the set of arguments and counterarguments a story character uses to make his individual choices on the basis of his preferences and attitudes. To reason explicitly about characters' value orderings, here we use [5]'s Extended Argumentation Frameworks (EAF) instead of VAFs, and the dialogue game of [6] to associate particular audiences with particular choices.

Narratives can be seen as actions giving rise to a sequence of state transitions. Action-based Alternating Transition Systems with Values (AATS+V) [1] provide the required features. An AATS comprises a set of states and transitions between them, with the transitions labelled with joint actions, that is actions comprising an action for each of the agents concerned. In an AATS+V, the transitions are also labelled with the values promoted and demoted that can be used to motivate the actions. A basic version of the parable of the Good Samaritan can be rendered as the AATS+V in Figure 1.

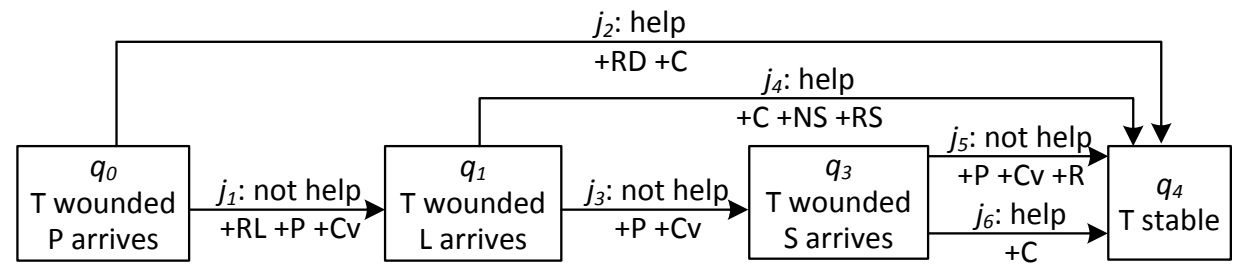

Figure 1. AATS + V for the Good Samaritan

In $q_{0}$, where the story begins, the traveller (T) is wounded. In $q_{4}$, the traveller's wounds have been bandaged and he is in a stable condition. In addition to the actions taken by the characters in the story $\left(j_{1}, j_{3}, j_{6}\right)$, we have also included some hypothetical 
actions the characters could have performed: for example, the Priest $(\mathrm{P})$ could also have helped the traveller $\left(j_{2}\right)$. Action choice in parables is often more or less binary (help or $\neg$ help, accept or $\neg$ accept in the Prodigal Son), so modelling these extra actions does not require much extra information besides the original story text. Values promoted (or demoted) are also included in the AATS+V: in Figure 1 we use Religious Duty (+RD), Religious Law (+RL), National Solidarity (+NS), Racial Solidarity (+RS), Compassion (+C), Prudence $(+\mathrm{P})$, Convenience $(+\mathrm{Cv})$ and Revenge $(+\mathrm{R})$. Adding the values requires more background knowledge. For example, we need to know that the traveller and the Levite (L) were of the same race, and that Samaritans (S) were seen as an enemy. Nowadays, this background information can be gained from Biblical texts, or from the many varied accounts on how parables should be interpreted, but it would have been well-known to the original audience. Part of the force of the story is that the two characters that pass by are people that the audience will be predisposed to admire, and so will attempt to find reasons that excuse their behaviour. The values in figure 1 are a selection that can motivate various reasons for not helping that the authors have heard from a variety of sources over the years. Note that the values are not themselves present in the story: they are used by audiences in their efforts to make sense of the behaviour of the characters involved.

Given the AATS $+\mathrm{V}$, we can construct the possible arguments for the different characters. The basic idea expressed in [1] is that the AATS+V serves as a formal grounding for arguments that instantiate the Practical Reasoning Argumentation Scheme (PRAS). Informally this is: in the current circumstances $R$ you should perform the action $A$ which will result in these new circumstances $S$, which will promote value $V . R$ and $S$ are states in the AATS $+\mathrm{V}, A$ is part of a joint action leading from $R$ to $S$, and $+V$ is a label on the corresponding transition. A formal version is given in [1]. Given this mapping of PRAS to an AATS+V, we can generate the arguments from the AATS $+\mathrm{V}$, noting that arguments for different actions attack each other because the actions are mutually exclusive, i.e., one cannot help and not help someone at the same time. First, there are the two arguments that might apply to the priest.

- $A_{1}$ : I should help the man because I have a religious duty to do so. This will promote Religious Duty (+RD)

- $A_{2}$ : I should not help the man because I risk uncleanliness through contact with his blood. This will promote Religious Law (+RL).

The following apply to the Levite as well as the Priest.

- $A_{3}$ : I should help the man because he is a fellow countryman. This will promote National Solidarity (+NS).

- $A_{4}$ : I should help the man because he is of my race. This will promote Racial Solidarity (+RS).

None of the above arguments apply to the Samaritan. The following arguments apply to all three characters.

- $A_{5}$ : I should help the man because he is a fellow human being. This will promote Compassion $(+\mathrm{C})$.

- $A_{6}$ : I should not help the man because it may be trap and I may be robbed. This will promote Prudence $(+\mathrm{P})$.

- $A_{7}$ : I should not help the man because it will interrupt my journey. This will promote Convenience $(+\mathrm{Cv})$. 
Although $A_{6}$ is based on a reason not explicit in the story, such traps were apparently common at the time, and would be likely to occur to the original audience as a reason why a good man might reasonably pass a wounded traveller by. In interpreting stories the background knowledge and beliefs of the audience need to be kept in mind. Finally there is an argument that applies only to the Samaritan:

- $A_{8}$ : I should not help this man, because his people have quarrelled with mine. This will promote Revenge $(+\mathrm{R})$.

Arguments $A_{1}-A_{4}$ relate to duties of one sort or another, arising from religious law or duty, or one form or another of social relationship (nation, race). $A_{5}-A_{8}$ all arise from natural human instincts, unconnected with any social institution.

From these arguments, we can construct a Value-based Argumentation Framework (VAF). The purpose of building a VAF is to find a subset of the arguments which is at once conflict free (i.e. no two arguments in the subset attack one another), and collectively able to defend itself (i.e. any attacker of an argument in the subset is itself attacked by an argument in the subset). The maximal such subset is called a preferred extension, and represents a maximal consistent position given the arguments presented. The key feature of VAFs is that they allow a distinction to be made between successful attacks (defeats) and unsuccessful attacks, on the basis of the values associated with the arguments and audience preferences between these values. Attacks succeed only if the value associated with the attacker is ranked equal to, or higher than, the argument it attacks. The VAF thus accounts for elements of subjectivity in that the arguments that are acceptable are dependent upon the audience's ranking of the values involved in the scenario.

We can now explain the actions of the three characters by considering the different value orderings of the different audiences. We may suppose that the Priest puts religion before all else (i.e., RD and RL are preferred to RS, NS, $\mathrm{Cv}, \mathrm{C}$ and $\mathrm{P}$ ). He has a conflict between $A_{1}$, that he should help to promote RD, and $A_{2}$, that he should not help to promote RL. In the story, he chooses to observe the law. This ranking of strict observance of the law over more human concerns is criticised elsewhere in the Gospels. The Levite must be supposed to act on either $A_{6}$ or $A_{7}$, overriding the specific duties of $A_{3}$ and $A_{4}$ as well as $A_{5}$. But because the Levite is a type of a morally respectable man, it must be assumed that we are being invited to conclude that these preferences are acceptable in the eyes of the current moral climate: that it is morally acceptable for prudence to override obligations arising from country or race, or from natural feelings of compassion. The Samaritan, in contrast has no duties prompting him help the man, and must balance his compassion against the other natural human instincts. That he helps the man $\left(A_{5}\right)$, can only be explained in terms of him putting compassion before all other values, individually and in combination. The lawyer concludes that this is what being a neighbour really is, and we are invited to do likewise.

\subsection{Stories as arguments in a dialogical context}

We now consider stories as arguments. We use the machinery of [5] to represent statements about value orderings as arguments in an Extended Argumentation Framework (EAF) to enable us to reason about which preferences will explain the behaviour of the various characters. As well as arguments and attacks between arguments, EAFs also contain a set of attacks on attacks. The idea is that preferences attack some attacks between 


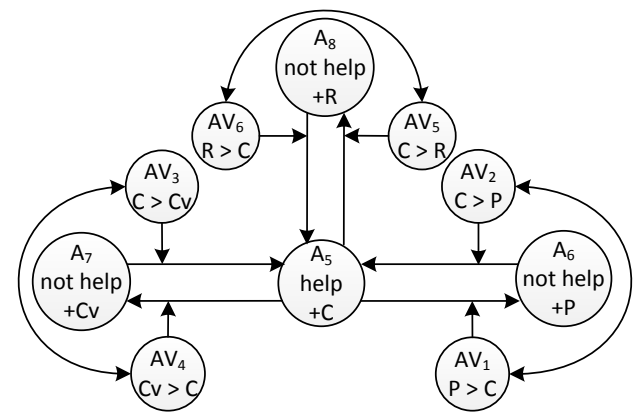

Figure 2. EAF for the Samaritan

arguments and thus determine which attacks succeed. In the EAF for the Samaritan there are potentially two value-preference arguments for each pair of values, for example: $A V_{1}$ : $P>C$; and $A V_{2}: C>P$.

These pairs will mutually attack, but more importantly they will attack the attack from the argument motivated by the less preferred value on arguments motivated by the other value. The complete EAF for the parable will now contain all the base arguments $A_{1}-A_{8}$ and arguments expressing the value preferences. We also introduce arguments for the various characters: $A C_{1}$ (Character is a priest), $A C_{2}$ (Character is a Levite) and $A C_{3}$ (Character is a Samaritan), to enable us to eliminate arguments which do not apply to particular characters: thus $A C_{1}$ will attack $A_{8}, A C_{2}$ will attack $A_{1}, A_{2}$ and $A_{8}$, and $A C_{3}$ will attack $A_{1}, A_{2}, A_{3}$ and $A_{4}$. Adding $A C_{3}$ to the AF that contains all characters' arguments $A_{1}-A_{8}$ thus produces the EAF applicable to just the Samaritan, shown in Figure 2. Similarly, $A C_{2}$ and $A C_{1}$ give the EAF applicable to the Levite and the priest respectively.

Having established appropriate EAFs for the various characters, we need to evaluate them to explain the choices they make. For the Samaritan, we need to construct an admissible set containing an argument to justify helping the traveller, and then to consider what value preferences it contains. One way to construct admissible sets from AFs is to use a dialogue game. A game for VAFs expressed as EAFs was given in [6]. First, the arguments of the EAF are rewritten as meta level statements. This is a purely mechanical process. Attacks from value based arguments can be rejected in two ways: not only can we reject the attacking argument, but also we can prefer the value of the attacked argument. Each pair of mutually attacking arguments can be rewritten as a regular AF; Figure 3 shows the new, regular $\mathrm{AF}$, structure for the pair of arguments $A_{5}$ and $A_{6}$.

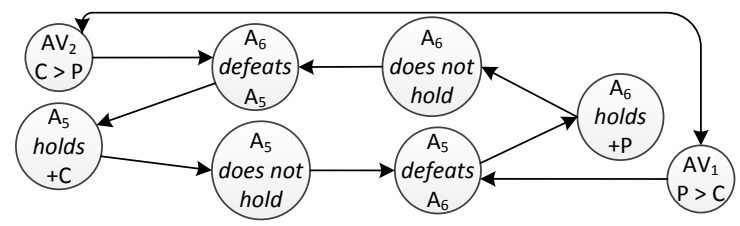

Figure 3. Regular $\mathrm{AF}$ for the $A_{5}-A_{6}$ part if the EAF in figure 2

The game proceeds by the proposer playing an argument, the opponent playing an attacker, the proposer playing an attacker of that argument and so on, until one player 
cannot move. At this point a player can back up to a choice point and play a different argument. This continues until no moves are possible (note that arguments under attack cannot be played). At this point we will have an admissible set containing the arguments played by the last player to move. If this was the proposer is will contain the original argument and this will have been shown to be acceptable. Because it is the Samaritan's preference we are trying to determine, we use the EAF in figure 2, rewritten as a regular AF (so that the arguments played are actually meta-arguments). The dialogue then proceeds as follows:

Samaritan: $A_{5}$ holds. This is an argument justifying what the Samaritan did in the story: current position is $\left\{A_{5}\right.$ holds $\}$.

Opponent: $A_{6}$ defeats $A_{5}$. Opponent chooses a way to attack $A_{5}$.

Samaritan: $A V_{2} \mathrm{C}>\mathrm{P}$. The preference argument is played: the alternative would eventually require $A_{5}$ holds to be played, but this is under attack. Current position is $\left\{A_{5}\right.$ holds, $\left.\mathrm{C}>\mathrm{P}\right\}$.

Opponent: $A_{7}$ defeats $A_{5}$. Opponent cannot play $P>C$, because it is under attack, and so backs up and chooses another line of attack.

Samaritan: $A V_{3} \mathrm{C}>\mathrm{Cv}$. Current position is $\left\{A_{5}\right.$ holds, $\left.\mathrm{C}>\mathrm{P}, \mathrm{C}>\mathrm{Cv}\right\}$.

Opponent: $A_{8}$ defeats $A_{5}$. Again the opponent must back up since $C v>C$ is under attack.

Samaritan: $A V_{5} \mathrm{C}>\mathrm{R}$. Current position is $\left\{A_{5}\right.$ holds, $\left.\mathrm{C}>\mathrm{P}, \mathrm{C}>\mathrm{Cv}, \mathrm{C}>\mathrm{R}\right\}$.

At this point the opponent must stop, since there are no further lines of attack. The Samaritan's position, $\left\{A C_{3}, A_{5}, A V_{2}, A V_{3}, A V_{5}\right\}$, comprises an argument justifying his action $\left(A_{5}\right)$ and the three value preferences required to defend that argument $\left(A V_{2}, A V_{3}\right.$ and $\left.A V_{5}\right)$. It is exactly this position that the audience is being urged to adopt, since it provides the answer to the lawyer's question "what does it mean to love your neighbour like yourself?".

\subsection{Implementing our model}

Once the story has been represented as an AATS $+\mathrm{V}$, implementation is straightforward. Here we assume the process of constructing the AATS to be manual, comparable to the construction of a knowledge base to support other structured argumentation systems. In fact constructing an AATS from a story is simpler than representing a factual problem, because the conventions of fiction are that things not mentioned in the story are not relevant, and so need not be represented in the AATS, allowing the propositions and actions required to be kept to a minimum. Representing an AATS requires: states: with attributes for state ID, and for each relevant proposition; transitions: with attributes for ID, source state, destination state, joint action, values promoted and values demoted and joint actions: with attributes for ID and one action for each agent This information can be represented as appropriate for the programming language used (e.g. as relations and their attributes roles in Prolog, or as tables in a database). We can now identify the arguments using a suitable (e.g. Prolog or SQL) query which, given a source state, will retrieve the joint actions which promote a value and from these identify the target state, the action each agent must perform, and the particular value promoted to provide an instantiation of PRAS. A similar query will produce counterexamples by identifying the transitions which demote rather than promote values. An implementation applicable to e-participation was described in [9]. 
Given the arguments, a story can be reasoned with in a variety of different ways. It could be presented as a web based tool for presenting and critiquing arguments, as in [9]. Or, if implemented in an existing multi-agent system, the arguments could be used to influence other software agents in their choice of action. Another interesting possibility is to allow humans to explore the stories and character motives through a dialogue with the computer. A generic framework for dialogue and an associated web interface (www . arg. dundee.ac.uk/arvina) have been developed [3] to allow users to engage in a dialogue about a set of arguments (such as instantiations of PRAS or an EAF) with software agents. Using this application it is possible to interrogate, for example, an agent representing the Samaritan about his motives, and thus gain a better understanding of the story. Furthermore, users can add arguments to challenge the interpretation of a story by arguing about, for example, the values represented in the AATS (see [1]'s critical questions for discussing the AATS).

\section{Conclusion}

In this paper we have shown how several current argumentation techniques (value based argumentation using AATS+V, EAFs and dialogues based on them) can be applied to story understanding, and how stories can be construed as arguments. In particular these techniques enable us to understand the story in terms of the practical reasoning used by the characters in making the choices depicted in the story. This in turn leads us to impute preferences to the characters, which we may adopt or reject according to how the story makes us feel about the various characters, enabling persuasion with respect to value preferences.

\section{References}

[1] K. Atkinson and T. Bench-Capon. Practical reasoning as presumptive argumentation using action-based alternation transition systems. Artificial Intelligence, 171(1015):855-874, 2007.

[2] F. Bex, T. Bench-Capon, and K. Atkinson. Did he jump or was he pushed? abductive practical reasoning. Artificial Intelligence and Law, 17:79-99, 2009.

[3] F. Bex, J. Lawrence, M. Snaith, and C. Reed. Implmenting the argument web. Communications of the ACM, 56(10):66-73, 2013.

[4] T. Govier and L. Ayers. Logic and parables: Do these narratives provide arguments? Informal Logic, 32(2):161-189, 2012.

[5] S. Modgil. Reasoning about preferences in argumentation frameworks. Artificial Intelligence, 173:901-934, 2009.

[6] S. Modgil and T. J. M. Bench-Capon. Integrating object and meta-level value based argumentation. In Proceedings of COMMA 2008, pages 240-251. IOS Press, 2008.

[7] E. Mueller. Understanding script-based stories using commonsense reasoning. Cognitive Systems Research, (4):307-340, 2004.

[8] R. Schank and R. Abelson. Scripts, Plans, Goals and Understanding: an Inquiry into Human Knowledge Structures. Lawrence Erlbaum, Hillsdale, NJ, 1977.

[9] M. Wardeh, A. Wyner, K. Atkinson, and T. Bench-Capon. Argumentation based tools for policy-making. In 14th International Conference on AI and Law, pages 249-250, 2013. 